\title{
Negative Energy Density States for the Dirac Field in Flat Spacetime
}

\author{
Dan N. Vollick \\ Department of Physics and Astronomy \\ University of Victoria \\ Victoria, British Columbia \\ P.O. BOX 3055 MS7700 \\ Canada \\ V8W 3P6
}

\begin{abstract}
Negative energy densities in the Dirac field produced by state vectors that are the superposition of two single particle electron states are examined. I show that for such states the energy density of the field is not bounded from below and that the quantum inequalities derived for scalar fields are satisfied. I also show that it is not possible to produce negative energy densities in a scalar field using state vectors that are arbitrary superpositions of single particle states.
\end{abstract}

\section{Introduction}

Recent work on wormholes [1, 2, 3] and the 'warp drive' [4] has generated interest in matter that violates the weak energy condition. Most discussions of such exotic matter occur within the context of quantum field theory and deal with bosonic fields [1, 2, 5, 6, 8, 9] (see [10, 11, 12] for a classical discussion and [7] for a discussion of 
fermionic fields in a curved spacetime). Recently Ford and Roman [8, 9] have shown that in flat spacetime the energy density of a massless scalar field and the electromagnetic field satisfy the quantum inequalities

$$
\hat{\rho} \equiv \frac{t_{0}}{\pi} \int_{-\infty}^{\infty} \frac{<: T_{00}:>}{\left(t^{2}+t_{0}^{2}\right)} d t \geq-\frac{A}{2 V} \sum_{k} \omega_{k} e^{-2 \omega_{k} t_{0}}
$$

in the finite volume case and

$$
\hat{\rho} \geq-\frac{3 A}{32 \pi^{2} t_{0}^{4}}
$$

in the infinite volume case, where $<: T_{00}:>$ is the expectation value of the normal ordered energy density, $A=1$ for a massless scalar field, and $A=2$ for the electromagnetic field. The quantity $\hat{\rho}$ samples $<: T_{00}:>$ over a time of order $t_{0}$. For simplicity I will refer to $<: T_{00}:>$ as the energy density of the field.

Unfortunately the methods used above to obtain general constraints on the energy densities cannot be applied to the Dirac equation. In this paper I will look at the negative energy densities that occur in states that are the superposition of two single particle electron states. For such states I show that the energy density is not bounded from below and that an observer at a fixed spatial point sees the energy density as a wave propagating by at the speed of light superimposed on a positive background. In certain regions of this wave the energy density is negative and in other regions it is positive. Thus the negative energy densities do not persist indefinitely. In fact, I will show that the negative energy density persists for a time that is inversely proportional to the minimum value of the energy density. I will also show that the quantum inequality (1) is satisfied for the states considered in this paper.

Finally I will show that, in contrast to the Dirac field, a scalar field cannot have negative energy densities for states that are arbitrary superpositions of single particle states.

Throughout this paper I will take $\hbar=c=1$ and the metric will be taken to have the signature $(-+++)$. 


\section{Negative Energy States for the Dirac Field}

The Lagrangian for the Dirac field is

$$
L=\frac{\imath}{2} \bar{\psi} \gamma^{\mu} \stackrel{\leftrightarrow}{\partial}_{\mu} \psi-m \bar{\psi} \psi
$$

Since the canonical energy momentum tensor $\theta^{\mu \nu}$ is not symmetric the Belinfante tensor 13

$$
\begin{aligned}
& T^{\mu \nu}=\theta^{\mu \nu}-\frac{\imath}{2} \partial_{\alpha}\left[\frac{\partial L}{\partial\left(\partial_{\alpha} \psi^{l}\right)}\left(J^{\mu \nu}\right)_{m}^{l} \psi^{m}+\frac{\partial L}{\partial\left(\partial_{\alpha} \psi^{l}\right)}\left(\bar{J}^{\mu \nu}\right)_{m}^{l} \bar{\psi}^{m}-\frac{\partial L}{\partial\left(\partial_{\mu} \psi^{l}\right)}\left(J^{\alpha \nu}\right)_{m}^{l} \psi^{m}\right. \\
& \left.-\frac{\partial L}{\partial\left(\partial_{\mu} \psi^{l}\right)}\left(\bar{J}^{\alpha \nu}\right)_{m}^{m} \bar{\psi}^{m}-\frac{\partial L}{\partial\left(\partial_{\nu} \psi^{l}\right)}\left(J^{\alpha \mu}\right)_{m}^{l} \psi^{m}-\frac{\partial L}{\partial\left(\partial_{\nu} \psi^{l}\right)}\left(\bar{J}^{\alpha \mu}\right)_{m}^{l} \bar{\psi}^{m}\right]
\end{aligned}
$$

should be used, where $J^{\mu \nu}$ is the generator of Lorentz transformations for $\psi$ and, $\bar{J}^{\mu \nu}$ is the generator of Lorentz transformations for $\bar{\psi}$. A short calculation gives

$$
T_{\mu \nu}=\frac{\imath}{4}\left[\bar{\psi} \gamma^{\mu} \stackrel{\leftrightarrow}{\partial}^{\nu} \psi+\bar{\psi} \gamma^{\nu} \stackrel{\leftrightarrow}{\partial}^{\mu} \psi\right]
$$

Thus

$$
T_{00}=\frac{\imath}{2}\left[\psi^{\dagger} \dot{\psi}-\dot{\psi}^{\dagger} \psi\right]
$$

Now define

$$
<\rho>=<: T_{00}:>
$$

and consider the Dirac field in a box of volume $V$. The field operator can be written in terms of creation and annihilation operators as

$$
\psi(x)=\sum_{k} \sum_{\alpha=1,2}\left[b_{\alpha}(k) u^{\alpha}(k) e^{\imath k \cdot x}+d_{\alpha}^{\dagger}(k) v^{\alpha}(k) e^{-\imath k \cdot x}\right]
$$

where

$$
\begin{aligned}
& u^{\alpha}(k)=\left(\begin{array}{l}
\sqrt{\frac{\omega+m}{2 \omega V}} \phi^{\alpha} \\
\frac{\vec{\sigma} \cdot \vec{k}}{\sqrt{2 \omega(\omega+m) V}} \phi^{\alpha}
\end{array}\right), \\
& v^{\alpha}(k)=\left(\begin{array}{l}
\frac{\vec{\sigma} \cdot \vec{k}}{\sqrt{2 \omega(\omega+m) V}} \phi^{\alpha} \\
\sqrt{\frac{\omega+m}{2 \omega V}} \phi^{\alpha}
\end{array}\right),
\end{aligned}
$$

$\phi^{1 \dagger}=(1,0)$, and $\phi^{2 \dagger}=(0,1)$. The creation and annihilation operators satisfy

$$
\left\{b_{\alpha}(k), b_{\alpha^{\prime}}^{\dagger}\left(k^{\prime}\right)\right\}=\delta_{\alpha, \alpha^{\prime}} \delta_{k, k^{\prime}}
$$


and

$$
\left\{d_{\alpha}(k), d_{\alpha^{\prime}}^{\dagger}\left(k^{\prime}\right)\right\}=\delta_{\alpha, \alpha^{\prime}} \delta_{k, k^{\prime}}
$$

with all other anticommutators vanishing. Substituting (8) into (7) gives

$$
\begin{aligned}
& <\rho>=\frac{1}{2} \sum_{k, k^{\prime}} \sum_{\alpha, \alpha^{\prime}}\left\{( \omega _ { k } + \omega _ { k ^ { \prime } } ) \left[<b_{\alpha}^{\dagger}(k) b_{\alpha^{\prime}}\left(k^{\prime}\right)>u^{\dagger \alpha}(k) u^{\alpha^{\prime}}\left(k^{\prime}\right) e^{-\imath\left(k-k^{\prime}\right) \cdot x}+\right.\right. \\
& \left.\left.<d_{\alpha^{\prime}}^{\dagger}\left(k^{\prime}\right) d_{\alpha}(k)>v^{\dagger \alpha}(k) v^{\alpha^{\prime}}\left(k^{\prime}\right) e^{\imath\left(k-k^{\prime}\right) \cdot x}\right]\right\}+\left(\omega_{k^{\prime}}-\omega_{k}\right)\left[<d_{\alpha}(k) b_{\alpha^{\prime}}\left(k^{\prime}\right)>v^{\dagger \alpha}(k) u^{\alpha^{\prime}}\left(k^{\prime}\right) e^{\imath\left(k+k^{\prime}\right) \cdot x}\right. \\
& \left.\left.-<b_{\alpha}^{\dagger}(k) d_{\alpha^{\prime}}^{\dagger}\left(k^{\prime}\right)>u^{\dagger \alpha}(k) v^{\alpha^{\prime}}\left(k^{\prime}\right) e^{-\imath\left(k+k^{\prime}\right) \cdot x}\right]\right\}
\end{aligned}
$$

where I have used : $d_{\alpha}(k) d_{\alpha^{\prime}}\left(k^{\prime}\right):=-d_{\alpha^{\prime}}^{\dagger}\left(k^{\prime}\right) d_{\alpha}(k)$.

Now consider a state vector of the form

$$
\mid \psi>=\frac{1}{\sqrt{1+\lambda^{2}}}\left[\left|k_{z}, 1>+\lambda\right| k_{x}, 2>\right]
$$

where $\left|k, \alpha>=b_{\alpha}^{\dagger}(k)\right| 0>$ and $\lambda$ is real. Since the state $\mid \psi>$ contains only electrons all expectation values in (13) containing $d_{\alpha}(k)$ or $d_{\alpha}^{\dagger}(k)$ vanish. Substituting (14) and (9) into (13) gives

$$
<\rho>=\frac{1}{\left(1+\lambda^{2}\right) V}\left[\omega_{k_{z}}+\lambda \beta+\lambda^{2} \omega_{k_{x}}\right]
$$

where

$$
\beta=\frac{k_{x} k_{z}\left(\omega_{k_{x}}+\omega_{k_{z}}\right) \cos \theta}{2 \sqrt{\omega_{k_{x}} \omega_{k_{z}}\left(\omega_{k_{x}}+m\right)\left(\omega_{k_{z}}+m\right)}}
$$

and $\theta=\left(k_{x}-k_{z}\right) x$. Note that $\langle\rho\rangle=\omega_{k_{z}} / V$ for $\lambda=0$ and $\langle\rho\rangle=\omega_{k_{x}} / V$ as $\lambda \rightarrow \infty$, as expected. It is easy to see that $\langle\rho\rangle$ will be negative if

$$
\beta^{2}>4 \omega_{k_{x}} \omega_{k_{z}}
$$

and if

$$
-\frac{\beta}{2}-\sqrt{\left(\frac{\beta}{2}\right)^{2}-\omega_{k_{x}} \omega_{k_{z}}}<\omega_{k_{x}} \lambda<-\frac{\beta}{2}+\sqrt{\left(\frac{\beta}{2}\right)^{2}-\omega_{k_{x}} \omega_{k_{z}}} .
$$

Consider the ultrarelativistic limit, $k_{x}, k_{z}>>m$. In this limit

$$
\beta=\frac{1}{2}\left(\omega_{k_{x}}+\omega_{k_{z}}\right) \cos \theta
$$

Equation (17) becomes

$$
\cos ^{2} \theta>\frac{16 \omega_{k_{x}} \omega_{k_{z}}}{\left(\omega_{k_{x}}+\omega_{k_{z}}\right)^{2}}
$$


For a solution to exist it is necessary that $16 \omega_{k_{x}} \omega_{k_{z}} \leq\left(\omega_{k_{x}}+\omega_{k_{z}}\right)^{2}$. This will be satisfied

if $\omega_{k_{x}} \geq(7+\sqrt{48}) \omega_{k_{z}}$ or if $\omega_{k_{z}} \geq(7+\sqrt{48}) \omega_{k_{x}}$. Thus it is possible to produce negative energy densities for state vectors of the form (14) if $\lambda$ is chosen to satisfy (18).

It is now easy to show that the energy density is not bounded from below. To simplify the discussion take $x^{\mu}=0$, so that $\cos \theta=1$. In the ultrarelativistic limit with $\omega_{k_{x}}>>\omega_{k_{z}}$

$$
\beta=\frac{1}{2} \omega_{k_{x}}
$$

and $-1 / 2 \leq \lambda \leq 0$. The energy density is given by

$$
<\rho>\simeq-\frac{\lambda \omega_{k_{x}}}{\left(1+\lambda^{2}\right) V}\left(\lambda+\frac{1}{2}\right) .
$$

Thus in the limit $\omega_{k_{x}} / V \rightarrow \infty$ the energy density at the spacetime point $x^{\mu}=0$ goes to $-\infty$, for $-1 / 2<\lambda<0$.

Now consider, within the above limits, the energy density on the spacetime. For general $x^{\mu}$

$$
<\rho>=\frac{\lambda \omega_{k_{x}}}{\left(1+\lambda^{2}\right) V}\left[\lambda+\frac{1}{2} \cos \left(\omega_{k_{x}}(t-x)\right)\right] .
$$

Thus $\langle\rho>$ is a cosine wave propagating at the speed of light superimposed on a positive background. The energy density at a fixed spatial point will be negative for a time $\Delta t$, which satisfies

$$
-V<\rho>_{\min } \Delta t=\frac{|\lambda|(1+2 \lambda)}{1+\lambda^{2}} \cos ^{-1}(2|\lambda|),
$$

where $\langle\rho\rangle_{\min }$ is the minimum value of $\langle\rho\rangle$ for fixed $\omega_{k_{x}}$ and $\lambda$. Since the wave propagates at the speed of light the extent of the negative energy density will satisfy the same expression as above with $\Delta t$ replaced by $\Delta x$. For large values of $|<\rho>| V$ (and $\lambda$ not too close to $-1 / 2$ or 0 ) the energy density will undergo rapid oscillations from positive to negative values. Note that the time average of the energy density is positive.

\section{Quantum Inequalities}

In this section I will show that the energy density satisfies the quantum inequality (1) for the state given in (14). To show this I will take the limit $m \rightarrow 0$ for the Dirac field. 
Substituting (23) into

$$
\hat{\rho}=\frac{t_{0}}{\pi} \int_{-\infty}^{\infty} \frac{<\rho>}{t^{2}+t_{0}^{2}} d t
$$

and taking $\vec{x}=0$ gives

$$
\hat{\rho}=\frac{\lambda \omega_{k_{x}}}{\left(1+\lambda^{2}\right) V}\left[\lambda+\frac{1}{2} e^{-\omega_{k_{x}} t_{0}}\right] .
$$

Thus $\hat{\rho}$ will be negative if

$$
\omega_{k_{x}} t_{0}<-\ln (2|\lambda|) .
$$

Now consider the quantum inequality (if) for $A=1$.

If periodic boundary conditions are imposed

$$
\omega=\frac{2 \pi}{L} \sqrt{n_{x}^{2}+n_{y}^{2}+n_{z}^{2}}
$$

where $n_{x}, n_{y}$, and $n_{z}$ are integers. Next note that

$$
\frac{1}{\sqrt{3}}\left(\left|n_{x}\right|+\left|n_{y}\right|+\left|n_{z}\right|\right) \leq \sqrt{n_{x}^{2}+n_{y}^{2}+n_{z}^{2}} \leq\left|n_{x}\right|+\left|n_{y}\right|+\left|n_{z}\right| .
$$

To see this define $f\left(n_{x}, n_{y}, n_{z}\right)$ by

$$
f\left(n_{x}, n_{y}, n_{z}\right)=\sqrt{n_{x}^{2}+n_{y}^{2}+n_{z}^{2}}-\alpha\left(\left|n_{x}\right|+\left|n_{y}\right|+\left|n_{z}\right|\right)
$$

where $\alpha$ is a positive constant. Now let

$$
\begin{aligned}
& \left|n_{x}\right|=r \cos \phi \sin \theta \\
& \left|n_{y}\right|=r \sin \phi \sin \theta \\
& \left|n_{z}\right|=r \cos \theta,
\end{aligned}
$$

where $0 \leq(\theta, \phi) \leq \pi / 2$. Thus $f$ can be written as

$$
f(r, \theta, \phi)=r(1-\alpha g(\theta, \phi))
$$

where

$$
g(\theta, \phi)=\sqrt{1+2 \sin ^{2}(\phi+\pi / 4)} \sin (\theta+\psi)
$$

and

$$
\cot (\psi)=\sqrt{2} \sin (\phi+\pi / 4) .
$$

Given that $0 \leq(\theta, \phi) \leq \pi / 2$, it is easy to see that $1 \leq g(\theta, \phi) \leq \sqrt{3}$. The function $g(\theta, \phi)$ has its maximum value of $\sqrt{3}$ when $\phi=\pi / 4$ and $\theta=\pi / 2-\cot ^{-1}(\sqrt{2})$. The 
minimum value of $g(\theta, \phi)$ occurs when $\theta=0$. In this case $g(0, \phi)=1$ for all $\phi$. The function $g(\theta, \phi)$ also equals 1 at $\theta=\pi / 2, \phi=0, \pi / 2$. Now if $\alpha=1$ in (30) then $f=r(1-g) \leq 0$. This gives the inequality $\sqrt{n_{x}^{2}+n_{y}^{2}+n_{z}^{2}} \leq\left|n_{x}\right|+\left|n_{y}\right|+\left|n_{z}\right|$. On the other hand, if $\alpha=1 / \sqrt{3}$ then $f=r(1-g / \sqrt{3}) \geq 0$. This gives the inequality $3^{-1 / 2}\left[\left|n_{x}\right|+\left|n_{y}\right|+\left|n_{z}\right|\right] \leq \sqrt{n_{x}^{2}+n_{y}^{2}+n_{z}^{2}}$. Thus (29) is proved.

To show that (1) is satisfied I will show that an even more restrictive inequality is satisfied. From (29)

$$
\omega_{k}^{-} \leq \omega_{k} \leq \omega_{k}^{+}
$$

where

$$
\omega_{k}^{-}=\frac{2 \pi}{\sqrt{3} L}\left[\left|n_{x}\right|+\left|n_{y}\right|+\left|n_{z}\right|\right]
$$

and

$$
\omega_{k}^{+}=\frac{2 \pi}{L}\left[\left|n_{x}\right|+\left|n_{y}\right|+\left|n_{z}\right|\right]
$$

Since

$$
\omega_{k}^{-} e^{-2 \omega_{k}^{+} t_{0}} \leq \omega_{k} e^{-2 \omega_{k} t_{0}}
$$

th quantum inequality (11) will be satisfied if

$$
\hat{\rho} \geq-\frac{1}{2 V} \sum_{k} \omega_{k}^{-} e^{-2 \omega_{k}^{+} t_{0}}
$$

is satisfied. Substituting in the expressions for $\omega_{k}^{+}$and $\omega_{k}^{-}$gives

$$
\hat{\rho} \geq-\frac{2 \sqrt{3} \pi}{L V}\left[\sum_{n=0}^{\infty} n e^{-\alpha n}\right]\left[2 \sum_{k=0}^{\infty} e^{-\alpha k}-1\right]^{2},
$$

where $\alpha=4 \pi t_{0} / L$. The sums can easily be performed, giving

$$
\hat{\rho} \geq-\frac{2 \sqrt{3} \pi}{L V} \frac{e^{-\alpha}\left(1+e^{-\alpha}\right)^{2}}{\left(1-e^{-\alpha}\right)^{4}} .
$$

Thus (11) will be satisfied if

$$
\frac{\lambda n_{x}}{1+\lambda^{2}}\left(\lambda+\frac{1}{2} e^{-\alpha n_{x} / 2}\right) \geq-\frac{\sqrt{3} e^{-\alpha}\left(1+e^{-\alpha}\right)^{2}}{\left(1-e^{-\alpha}\right)^{4}} .
$$

is satisfied. For $\lambda$ outside the interval $\left(-\frac{1}{2} e^{-\alpha n_{x} / 2}, 0\right)$ the above inequality will obviously be satisfied. Thus consider $-\frac{1}{2} e^{-\alpha n_{x} / 2}<\lambda<0$. Equation (42) will be satisfied if

$$
|\lambda| n_{x}\left(\lambda+\frac{1}{2} e^{-\alpha n_{x} / 2}\right) \leq \frac{\sqrt{3} e^{-\alpha}\left(1+e^{-\alpha}\right)^{2}}{\left(1-e^{-\alpha}\right)^{4}}
$$


is satisfied. Now let $\lambda=-\frac{\sigma}{2} e^{-\alpha n_{x} / 2}$. The above inequality becomes

$$
\frac{1}{4} \sigma n_{x} e^{-\alpha n_{x}}(1-\sigma) \leq \frac{\sqrt{3} e^{-\alpha}\left(1+e^{-\alpha}\right)^{2}}{\left(1-e^{-\alpha}\right)^{4}} .
$$

The left hand side is maximized for $\sigma=1 / 2$ and for $n_{x}=1 / \alpha$. But $n_{x}$ is a positive integer. Thus for $\alpha \geq 1$ take $n_{x}=1$. The above inequality will then be satisfied (for $\alpha \geq 1)$ if

$$
\frac{1}{16} \leq \frac{\sqrt{3}\left(1+e^{-\alpha}\right)^{2}}{\left(1-e^{-\alpha}\right)^{4}}
$$

is satisfied. This is obviously satisfied for all $\alpha \geq 1$. For $\alpha<1$ let $n_{x}=\frac{1}{\alpha}$ (i.e. generalize $n_{x}$ to a real number). The inequality (44) will be satisfied if

$$
\frac{1}{16} e^{-1} \leq \frac{\sqrt{3} \alpha e^{-\alpha}\left(1+e^{-\alpha}\right)^{2}}{\left(1-e^{-\alpha}\right)^{4}}
$$

is satisfied. In the interval $0<\alpha<1$ the right hand side is a monotonically decreasing function of $\alpha$ with a minimum value of $e^{-1}\left(1+e^{-1}\right)^{2}\left(1-e^{-1}\right)^{-4}$. Thus the above inequality is satisfied. Therefore inequality (42) will be satisfied, which implies that the quantum inequality (1) will be satisfied.

\section{The Klein-Gordon Field}

In this section I will show that the energy density for a massive scalar field is positive for all states that are arbitrary superpositions of single particle states.

The scalar field operator can be written in terms of creation and annihilation operators as

$$
\phi(x)=\imath \sum_{k} \frac{1}{\sqrt{2 V \omega_{k}}}\left(a_{k} e^{\imath k^{\mu} x_{\mu}}-a_{k}^{\dagger} e^{-\imath k^{\mu} x_{\mu}}\right) .
$$

The energy-momentum tensor for the scalar field is given by

$$
T^{\mu \nu}=\partial^{\mu} \phi \partial^{\nu} \phi-\frac{1}{2} \eta^{\mu \nu}\left(\partial_{\alpha} \phi \partial^{\alpha} \phi+m^{2} \phi^{2}\right) .
$$

A short calculation gives

$$
<\rho>=\frac{1}{2 V} R e \sum_{k, k^{\prime}} \frac{\left(\omega_{k} \omega_{k^{\prime}}+\vec{k} \cdot \vec{k}^{\prime}+m^{2}\right)}{\sqrt{\omega_{k} \omega_{k^{\prime}}}}<a_{k^{\prime}}^{\dagger} a_{k}>e^{\imath\left(k^{\mu}-k^{\prime} \mu\right) x_{\mu}}
$$




$$
+\frac{\left(\omega_{k} \omega_{k^{\prime}}+\vec{k} \cdot \overrightarrow{k^{\prime}}-m^{2}\right)}{\sqrt{\omega_{k} \omega_{k^{\prime}}}}<a_{k^{\prime}} a_{k}>e^{\imath\left(k^{\mu}+k^{\prime} \mu\right) x_{\mu}}
$$

The state vector can be written as

$$
\left|\psi>=\frac{1}{N} \sum_{k} \alpha_{k}\right| k>
$$

where the $\alpha_{k}$ are arbitrary complex numbers and $N$ is chosen so that $|\psi\rangle$ is normalized. The energy density can now be written as

$$
<\rho>=\frac{1}{2 V N^{2}} \sum_{k, k^{\prime}} \frac{\left(\omega_{k} \omega_{k^{\prime}}+\vec{k} \cdot \vec{k}^{\prime}+m^{2}\right)}{\sqrt{\omega_{k} \omega_{k^{\prime}}}} \alpha_{k^{\prime}}^{*} \alpha_{k} e^{\imath\left(k^{\mu}-k^{\prime \mu}\right) x_{\mu}} .
$$

Now define

$$
\beta=\sum_{k} \sqrt{\omega_{k}} \alpha_{k} e^{\imath k^{\mu} x_{\mu}}, \quad \lambda=m \sum_{k} \frac{\alpha_{k}}{\omega_{k}} e^{\imath k^{\mu} x_{\mu}}
$$

and

$$
\vec{\gamma}=\sum_{k} \frac{\vec{k}}{\sqrt{\omega_{k}}} \alpha_{k} e^{\imath k^{\mu} x_{\mu}}
$$

Thus for sate vectors of the form (51)

$$
<\rho>=\frac{1}{2 V N^{2}}\left(|\beta|^{2}+|\lambda|^{2}+|\vec{\gamma}|^{2}\right)
$$

and the energy density is non-negative.

\section{Conclusion}

In this paper I examined the negative energy densities that can be produced in the Dirac field by state vectors of the form

$$
\mid \psi>=\frac{1}{\sqrt{1+\lambda^{2}}}\left(\left|k_{z}, 1>+\lambda\right| k_{x}, 2>\right)
$$

where $\mid k_{z}, 1>$ and $\mid k_{x}, 2>$ are single particle electron states and $\lambda$ is real. I showed that if $k_{x}, k_{z}>>m$, the energy density at a space-time point $x^{\mu}$ will be negative if $\lambda$ is chosen so that

$$
-\frac{\beta}{2}-\sqrt{\left(\frac{\beta}{2}\right)^{2}-\omega_{k_{x}} \omega_{k_{z}}}<\omega_{k_{x}} \lambda<-\frac{\beta}{2}+\sqrt{\left(\frac{\beta}{2}\right)^{2}-\omega_{k_{x}} \omega_{k_{y}}}
$$


is satisfied, where

$$
\beta=\frac{1}{2}\left(\omega_{k_{x}}+\omega_{k_{z}}\right) \cos \left[\left(k^{\mu}-k^{\prime \mu}\right) x_{\mu}\right]
$$

Since I am taking $\lambda$ to be real it is necessary that $\beta \geq 4 \omega_{k_{x}} \omega_{k_{z}}$. This will be satisfied if $\omega_{k_{x}} \geq(7+\sqrt{48}) \omega_{k_{z}}$ or if $\omega_{k_{z}} \geq(7+\sqrt{48}) \omega_{k_{x}}$.

If, in addition to $k_{x}, k_{z}>>m$, one takes $\omega_{k_{x}}>>\omega_{k_{z}}$ then

$$
-\frac{1}{2} \leq \lambda \leq 0, \quad \beta=\frac{1}{2} \omega_{k_{x}}
$$

and

$$
<\rho>=\frac{\lambda \omega_{k_{x}}}{\left(1+\lambda^{2}\right) V}\left(\lambda+\frac{1}{2}\right)
$$

at the point $x^{\mu}=0$. Thus $\left\langle\rho>\rightarrow-\infty\right.$ as $\omega_{k_{x}} / V \rightarrow \infty$, for $-1 / 2<\lambda<0$ and $<\rho>$ is not bounded from below.

An observer will see $\langle\rho>$ as a cosine wave propagating at the speed of light superimposed on a positive background. The time average of $\langle\rho\rangle$ is positive and the energy density will be negative for a time interval $\Delta t$, which satisfies

$$
-V<\rho>_{\min } \Delta t=\frac{|\lambda|(1+2 \lambda)}{1+\lambda^{2}} \cos ^{-1}(2|\lambda|)
$$

I also showed that the quantum inequality

$$
\hat{\rho} \equiv \frac{t_{0}}{\pi} \int_{-\infty}^{\infty} \frac{<\rho>d t}{t^{2}+t_{0}^{2}} \geq-\frac{1}{2 V} \sum_{k} \omega_{k} e^{-2 \omega_{k} t_{0}},
$$

which is satisfied by a massless scalar field, is satisfied by the Dirac field for state vectors of the form (56) in the limit $m \rightarrow 0$. Finally, I showed that, in contrast to the Dirac field, it is not possible to produce negative energy densities in a scalar field using state vectors that are arbitrary superpositions of single particle states.

\section{References}

[1] M.S. Morris and K.S. Thorne, Am. J. Phys. 56, 395 (1988)

[2] M.S. Morris, K.S. Thorne, and U. Yurtserver, Phys. Rev. Lett. 61, 1446 (1988)

[3] V.P. Frolov and I.D. Novikov, Phys. Rev. D42, 1057 (1990) 
[4] M. Alcubierre, Class. Quant. Grav. 11, L73 (1994)

[5] H. Epstein, V. Glaser, and A. Jaffe, Nouvo Cimento 36, 1016 (1965)

[6] C.-I. Kuo, Nouvo Cimento 112B, 629 (1997)

[7] J.B. Griffiths, J. Phys. A 12, 2429 (1979)

[8] L.H. Ford and T.A. Roman, Phys. Rev. D51, 4277 (1995)

[9] L.H. Ford and T.A. Roman, Phys. Rev. D55, 2082 (1997)

[10] D.N. Vollick, Phys. Rev. D56, 4720 (1997)

[11] D.N. Vollick, Phys. Rev. D56, 4724 (1997)

[12] D.N. Vollick, Phys. Rev. D52, 3576 (1995)

[13] F. Belinfante, Physica 6, 887 (1939) 